\title{
A Forecasting System of Carbon Price in the Carbon Trading Markets Using Artificial Neural Network
}

\author{
Ming-Tang Tsai and Yu-Teing Kuo
}

\begin{abstract}
In this paper, a carbon price forecasting system is proposed to quickly and accurately predict the carbon price for participants. The data including the carbon trading price, oil price, coal price and gas price are first calculated and the data clusters are embedded in the Excel Database. Based on the Radial Basis Function Network (RBFN) and Ant Colony Optimization (ACO), an Ant-Based Radial Basis Function Network (ARBFN) is constructed in the searching process. The optimal parameters obtained from the ACO enable the learning rate parameters to regulate and improve the predicting errors during the training process. By linking the ARBFN and Excel database, the training stages of the ARBFN retrieve the input data from the Excel Database so that the efficiency and accuracy of the predicting system can be analyzed. A comparison of the Back-propagation Neural Network (BPN), Radial Basis function (RBFN), Probability Neural Network (PNN) and the ARBFN show that the converging solution is obtained by the prediction process. Simulation results will provide an accurate and real-time method for participants to forecast carbon price and raise the market competition in a carbon trading market.
\end{abstract}

Index Terms - Carbon trading market, radial basis function network, ant colony optimization, carbon price.

\section{INTRODUCTION}

Since global warming is becoming more and more serious, controlling greenhouse gases is now recognized as a serious issue. A study by the Intergovernmental Panel on Climate Change (IPCC) noted that the main greenhouse gas is $\mathrm{CO} 2$ gas [1]. Furthermore, the United Nations Framework Convention on Climate Change (UNFCCC) announced [2] that the Carbon and Cap-and-Trade concept will be applied to enforce the reduction of carbon emissions. In the carbon trading market, an important corollary factor is the carbon price prediction.

The European Union (EU) Emission Trading Scheme (EU-ETS) is commonly recognized by the carbon trading market as one of the most successful market models [3]. The EU-ETS that operates the market is coordinated to competitively determine the carbon price. Many factors, including historical carbon prices, but also oil, coal, and gas prices could impact the carbon price at various times of the month, season or year, according to the status of the different industry sectors [4], [5]. The energy prices and carbon prices in the wholesale market are mutually intertwined activities, and prices could rise to a hundred times their normal value as

Manuscript received December 5, 2012; revised February 8, 2013.

Ming-Tang Tsai and Yu-Teing Kuo are with the Department of Electrical Engineering, Cheng-Shiu University, Kaohsiung, Taiwan, R.O.C (e-mail: tsaymt@csu.edu.tw). a reflection of volatility. Although there are a few prediction methods for carbon prices [6], [7], prediction is difficult due to the nonlinearity and complexity of the market data.

Recently, the Artificial Neural Network (ANN) has been applied to forecast prices in various fields [8]-[11]. ANN is a simple, powerful and flexible tool for forecasting; it provides better solutions for modeling complex non-linear relationships than the traditional linear models do. Among ANNs, the Radial Basis Function Network (RBFN) can function as a classifier and forecaster, and it has the advantage of being a simple construct [12]. However, the RBFN probably causes over-learning because of its large adjustable parameters. In this paper, Ant Colony Optimization (ACO) [13] is applied to adjust these parameters in the learning process of RBFN. ACO can apply the activity characteristics of biotic populations to optimization problems [14]. The advantages of the ACO algorithm are that individual solutions within a range of possible solutions can converge to the optimal solution, and the algorithm has a faster convergence rate. Combining the ACO and neural network has proven successful in optimal searching and engineering problems [15]-[17].

Performing carbon price forecasting is complicated, especially when trying to find the best strategy in a world of uncertainties. Accurate carbon price estimations may help industries to effectively plan their future investments through the carbon trading mechanism. The benefit of carbon trading is huge revenuesz generated by the carbon market [18], [19]. To decide on the appropriate carbon handling price, it is necessary to accurately predict the day-head carbon price. In this paper, an ACO-based RBFN (ARBFN) is proposed to improve the searching ability. This paper develops season-ahead forecasts for carbon price using ARBFN. The Mean Absolute Percentage Error (MAPE), Mean Absolute Error (MAE) and Root Mean Square Error (RMSE) obtained from the forecasting results demonstrate that ARBFN can efficiently forecast the carbon price for any season of the year.

\section{ANT-BASED RADIAL BASIS FUNCTION NETWORK}

ARBFN consists of the input, hidden, and output layers. The unknown j-th input vector $X_{j}=\left[x_{j 1}, x_{j 2}, \ldots, x_{j i}, \ldots, x_{j N}\right]$, $i=1,2, \ldots, N, j=1,2, \ldots, M$, is connected to the input layer. The number of output nodes $y_{j}, j=1,2, \ldots, M$, is equal to the number of training input-output data pairs, that is to said, input nodes and output nodes are paired. The $\mathrm{j}$-th hidden nodes vector $H_{j}=\left[H_{j 1}, H_{j 2}, \ldots, H_{j k}, \ldots, H_{j K}\right], k=1,2, \ldots, K, j=1$, $2, \ldots, M$. The weights $w_{j k}$ connecting the $k$-th hidden node with the $j$-th output node. The ARBFN structure is shown in 
Fig. 1, the "ACO" process is performed in the adjusting error stage.

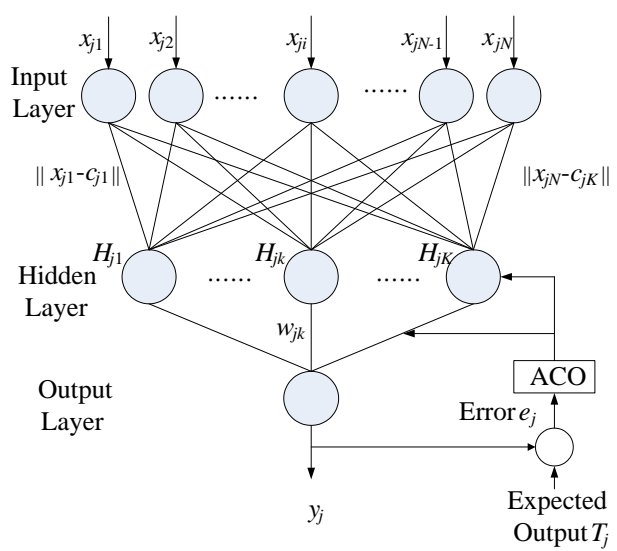

Fig. 1. The ARBFN structure $j$-th forecast

\section{A. Input Layer}

In this paper, $x_{i}$ is the $i$-th variable of the expected output. For each training data pairs, set input matrix $X=\left[x_{j i}\right]_{M \times N}, j=1$, $2, \ldots, M, i=1,2, \ldots, N$.

\section{B. Hidden Layer}

In the hidden layer, $C_{j}=\left[c_{j 1}, \ldots, c_{j k}, \ldots, c_{j K}\right]$ is called the $j$-th center of ARBFN. $\left\|x_{j i}-c_{j k}\right\|$ is the Euclidean distance between the $i$-th node of the input layer and the $k$-th node of hidden layer. The Euclidean distance is determined by Eq. (1) The $k$-th hidden layer output is defined as Eq. (2)

$$
\begin{gathered}
\left\|x_{j i}-c_{j k}\right\|=\sqrt{\sum_{i=1}^{N}\left(x_{j i}-c_{j k}\right)^{2}} \\
H_{j k}=\varphi_{j k}\left(\sqrt{\sum_{i=1}^{N}\left(x_{j i}-c_{j k}\right)^{2}}\right) \\
\varphi(x)=e^{-x^{2} / \sigma^{2}}
\end{gathered}
$$

where Eq. (3) the function $\varphi(\bullet)$ is Gaussian distribution function and $\sigma$ is smoothing parameter.

\section{Output Layer}

In the output layer, let $w_{j k}$ be the weight between hidden node $H_{j k}$ and output node $y_{j}$ and the $j$-th output of output layer be as Eq. (4).

$$
y_{j}=\sum_{k=1}^{K} w_{j k} H_{j k}
$$

\section{Stochastic Gradient Approach (SGA) [20]}

In order to adjust three parameters, which are weights $w_{j k}$, the center of $\mathrm{C}$ and the smoothing parameters $\sigma_{j k}$ of function $\varphi(\bullet)$, Stochastic Gradient Approach (SGA) is adopted. SGA process is:

1) Calculate Euclidean distance $\left\|x_{j i}-c_{j k}\right\|$,

2) Calculate hidden layer output $H_{j k}$ by Eq. (2),

3) Calculating output layer output by Eq. (4),

4) Calculate the error between simulation output $y_{j}$ and its expected value $T_{j}$ by error function. The related parameters are updated by Eq.(6), Eq.(7) and Eq.(8)

$$
\begin{gathered}
w_{j k}(n+1)=w_{j k(n)}-\mu_{j w} \frac{\partial}{\partial w_{j k}} e_{j}(n) \\
c_{j k}(n+1)=c_{j k(n)}-\mu_{j c} \frac{\partial}{\partial c_{j k}} e_{j}(n) \\
\sigma_{j k}(n+1)=\sigma_{j k(n)}-\mu_{j \sigma} \frac{\partial}{\partial \sigma_{j k}} e_{j}(n)
\end{gathered}
$$

\section{E. Ant Colony Optimization (ACO) Process}

The optimization method ACO is used to optimize three learning rates parameters: $\mu_{i w}, \mu_{i c}$ and $\mu_{i \sigma}$. There are seven steps to optimizing the learning rate parameters in the ACO optimization process:

Step 1: Set the number of ants ( $p$ ), the pheromone intensity $\left(\tau_{i j}\right)$, and consciousness constant $\left(\eta_{i j}\right)$, for each learning rate parameter.

Step 2: The initial solution is obtained by assigning a binary digit for the learning rate parameters, as shown in Fig. 2. To avoid an ant repeatedly passing the same place, the optimization process gives each ant $(p)$, its own path at time $i$ so that it can memorize where the ant has gone; $\tau_{i j}$ and $\eta_{i j}$ are used to control the ant's direction when moving to another place.

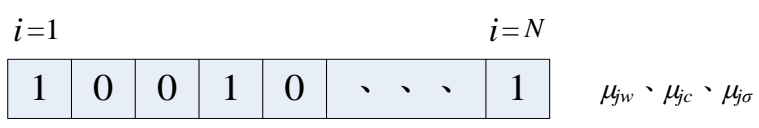

Fig. 2. A binary digit for the learning rate parameters

A matrix is designed to represent the ants' path $\left(\operatorname{tabu} u^{p}(i)\right)$. The ants' path matrix $\left(\operatorname{tabu}^{p}(i)\right)$ is defined as shown in Fig. 3 . Setting the learning rate parameters of ants as they traverse a selected path involves calculating the fitness values of all ants until a specified training accuracy is achieved. A sample is used to describe the basic concept of the ants' matrix, as shown in Fig. 3. If an ant of $\mu_{j w}$ parameter is located at array $(2,1)$ after the fitness value is calculated, the digital binary is set to 1 because the fitness value at array $(2,1)$ is best along this stage. The digital binary of array $(1,1)$ will be then set to 0 . When the ant passes array $(1,3)$, the digital binary of array $(2,3)$ will be set to 0 . The ants of $\mu_{j c}$ and $\mu_{j \sigma}$ parameters traverse their paths simultaneously. A new path matrix is generated when the ants have traversed the entire path.

Step 3: Calculate the probability of the ant's path. The state transition rule of the ant's path matrix used by ACO is given by Eq. (9), which gives the probability of ant $p$ at $\operatorname{array}(i, j)$ :

$$
P_{i j}^{p}(t)= \begin{cases}\frac{\alpha \cdot \tau_{i j}^{p}(t)+(1-\alpha) \cdot \eta_{i j}^{p}}{\sum_{l \notin t a b u^{p}}\left(\alpha \cdot \tau_{i l}^{p}(t)+(1-\alpha) \cdot \eta_{i j}^{p}\right.} & , j \notin t a b u^{p} \\ 0 & , \text { otherwise }\end{cases}
$$


$0<\alpha<1$ is a pheromone decay parameter; $\tau_{i j}^{p}(t)$ is the pheromone intensity at time $t$; and $\eta_{i j}^{p}(t)$ is the pheromone awareness at time $t$.

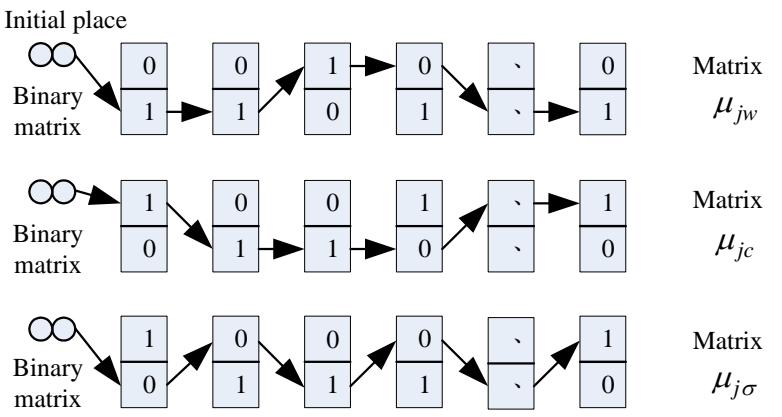

Fig. 3. The basic concept of the ants' matrix

Step 4: Generate the new binary matrix and calculate the ants' learning: $\mu_{j w}(t+1), \mu_{j c}(t+1)$, and $\mu_{j \sigma}(t+1)$.

Step 5: Calculate the output error of the fitness function. Step 6: Update the pheromone intensity:

$$
\tau_{i j}^{p}(t+1)=\rho \tau_{i j}^{p}(t)+\Delta \tau_{i j}^{p}
$$

$\rho$ is the constant of pheromone intensity $(0 \leq \rho \leq 1)$ and $\Delta \tau_{i j}^{p}$ is the deviation of pheromone intensity, as shown in Eq. (11):

$\Delta \tau_{i j}^{p}=\left\{\begin{array}{cl}Q / e_{i j}^{p} & , \text { the path }(i, j) \text { for } p-t h \text { ant } \\ 0 & , \text { other }\end{array}\right.$

$\mathrm{Q}$ is the released rate of pheromone $(0 \leq Q \leq 1)$ and $e_{i j}^{p}$ is the path error $(\mathrm{i}, \mathrm{j})$ for the $\mathrm{p}$-th ant.

Step 7: Implement the stopping rule. The error of fitness function is set to 0.00001 as the stopping criterion. If the convergent condition is not met, Step 2 to Step 6 will be rerun again.

\section{IMPLEMENTATION OF ARBFN}

Carbon price profiles are similar in different typoes. The fluctuation of carbon price may be due to the oil price, coal price, or gas price. ARBFN is capable of coping with complicated interactions between these three factors and the carbon price. Hence, these factors can be taken into account as inputs to the ARBFN.

The input layer contains three input variables: oil price, coal price and gas price. The hidden layer contains $K$ hidden nodes. The output layer only contains one output variable, the carbon price. ARBFN training is done to minimize the fitting error for a sample training setAfter the training procedure, the $t$-th hr forecasting data is input in the $t$-th hr ARBFN network to forecast the $\mathrm{t}$-th carbon price.

The price profile presents seasonal characteristics, usually season cycles. The cycles for the training data are classified as spring (January to March), summer (April to June), fall (July to September), and winter (October to December). A selection of similar season cycles for each year is trained in
ERBFN. For example, 7 similar cycles are selected for training to predict the carbon price in spring, summer, fall and winter. One season is taken to reflect similar days to the test data. The sample data of the European Climate Exchange (ECE) [4] are constructed in EXCEL Workspace. The data analysis and data storage can be easily manipulated with this database. To evaluate the forecasting accuracy of the ARBFN, the study uses the Mean Absolute Percentage Error (MAPE), Mean Absolute Error (MAE) and Root Mean Square Error (RMSE). The MAPE, MAE and RMSE are defined as:

$$
\begin{gathered}
M A P E=\frac{1}{T} \sum_{t=1}^{T} \frac{\left|P_{t}^{\text {ture }}-P_{t}^{\text {predict }}\right|}{P_{t}^{\text {ture }}} \times 100 \% \\
M A E=\frac{1}{T} \sum_{t=1}^{T}\left|P_{t}^{\text {ture }}-P_{t}^{\text {predict }}\right| \\
R M S E=\sqrt{\frac{1}{T} \sum_{t=1}^{T}\left|P_{t}^{\text {ture }}-P_{t}^{\text {predict }}\right|^{2}}
\end{gathered}
$$

where $P_{t}^{\text {ture }}$ is the actual price at hour $t ; P_{t}^{\text {predict }}$ is the forecasting price at $\mathrm{hr} t$; and $T$ is the number of testing data. Fig. 4 shows the forecasting procedure of ARBFN.

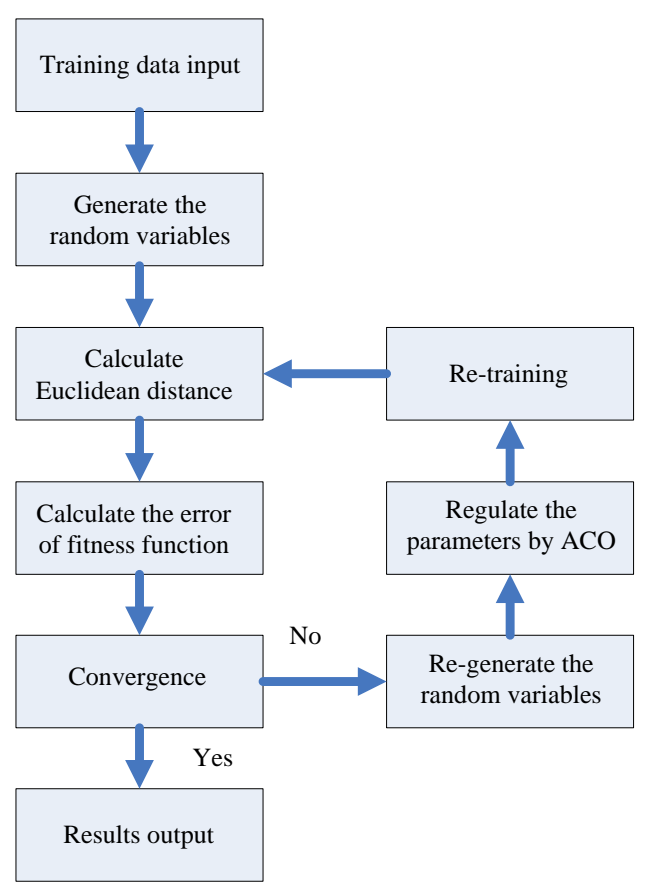

Fig. 4. The forecasting procedure of ARBFN

\section{RESUlts ANALysis}

The data from the ECE were used to train and test the proposed method. For comparison purposes, ARBFN, RBFN, PNN and BPN were also constructed for tests. The data set was divided into two parts: the training data and testing data, as shown in Table I. The training data were used for training and updating the biases and learning rates. The test data were used to test the proposed methods after training. The simulation was implemented with Matlab on a PIV-2.6GHZ computer with 512MB RAM. 


\section{A. Simulation Results}

Fig. 5 shows the season-ahead price forecasting for Case 1 . The forecasting results are compared with the actual carbon price. The MAPE, RMSE and MAE of ARBFN are 7.340\%, $12.13 \%$ and 0.1589 , respectively. The forecasting results of ARBFN can very closely track the actual carbon price with ACO applied. Note that the algorithm can even track the first spike well, which other algorithms cannot easily do. Common neural networks such as RBPN and BPN do not have this capability.

\begin{tabular}{|c|c|c|c|}
\hline Case & Training Data & $\begin{array}{c}\text { Training Data } \\
\text { (year/date) }\end{array}$ & Test data (Date) \\
\hline 1 & $\begin{array}{c}\text { The similar season of } \\
\text { Summer }\end{array}$ & $\begin{array}{c}2003 \sim 2009 \\
04.01 \sim 06.30\end{array}$ & $\begin{array}{c}2010.04 .01 \\
\sim \\
2010.06 .30\end{array}$ \\
\hline 2 & $\begin{array}{c}\text { The similar season of } \\
\text { Winter }\end{array}$ & $\begin{aligned} 2003 & \sim 2009 \\
10.01 & \sim 12.31\end{aligned}$ & $\begin{array}{c}2010.10 .01 \\
\sim \\
2010.12 .31\end{array}$ \\
\hline 3 & $\begin{array}{c}\text { The similar month of } \\
\text { Spring }\end{array}$ & $\begin{array}{c}2003 \sim 2009 \\
03.01 \sim 03.31\end{array}$ & $\begin{array}{c}2010.03 .01 \\
\sim \\
2010.03 .31\end{array}$ \\
\hline 4 & The similar month of Fall & $\begin{aligned} 2003 & \sim 2019 \\
10.01 & \sim 10.31\end{aligned}$ & $\begin{array}{c}2010.10 .01 \\
\sim \\
2010.10 .31\end{array}$ \\
\hline
\end{tabular}

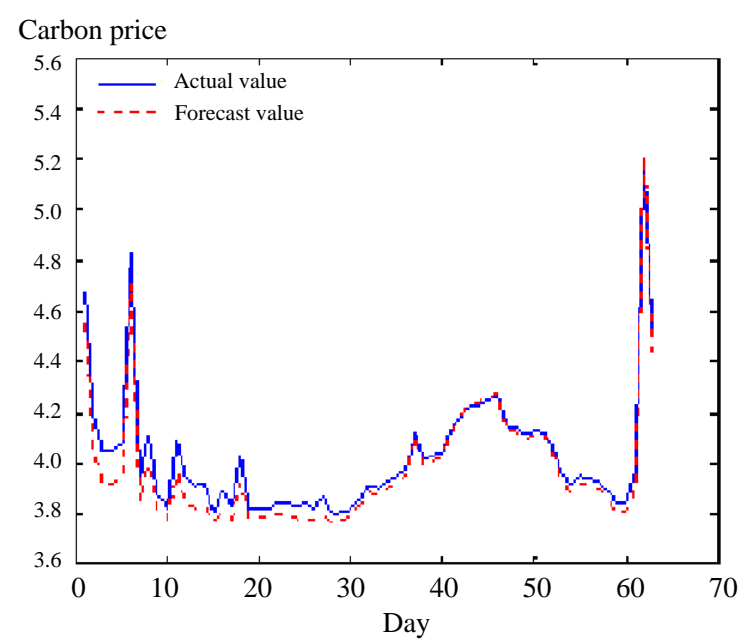

Fig. 5. Actual and forecast carbon price of case 1

Similarly, carbon price forecasting for Case 2 is shown in Fig. 6, where the MAPE value is about $6.60 \%$ for the ARBFN method. According to the above results, it is obvious that ARBFN has the ability to find better solutions.

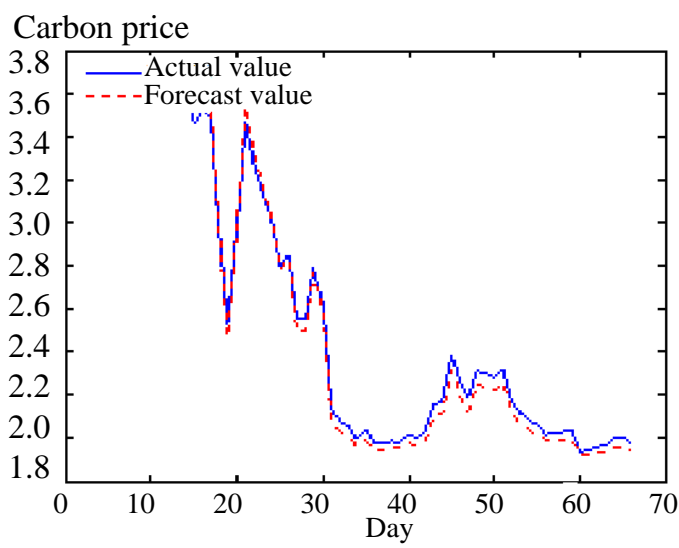

Fig. 6. Actual and forecast carbon price of case 2

Fig. 7 and Fig. 8 show the carbon price forecast for cases 3 and 4 , respectively. Case 4 is a typical fall month and the forecasting price of ARBFN is close to the actual carbon price values shown in Fig. 8. It can be seen that ERBFN has the capability to follow the spikes. The MAPE from the ARBFN method is about $9.180 \%$. Table II shows the RMSE, MAE and MAPE for all study cases.

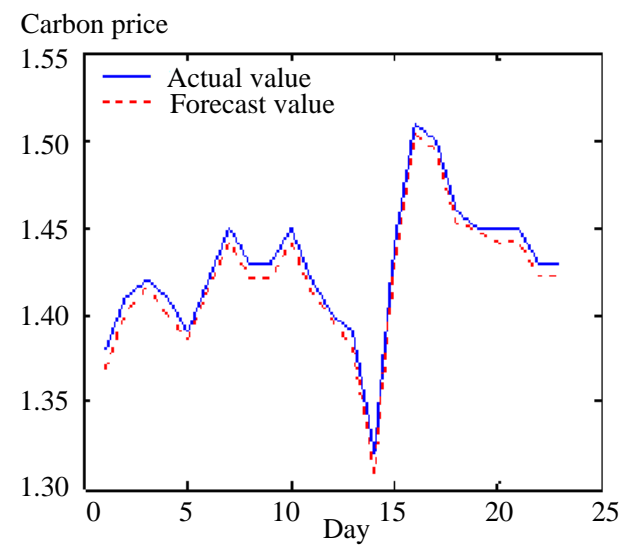

Fig. 7. Actual and forecast carbon price of case 3

Carbon price

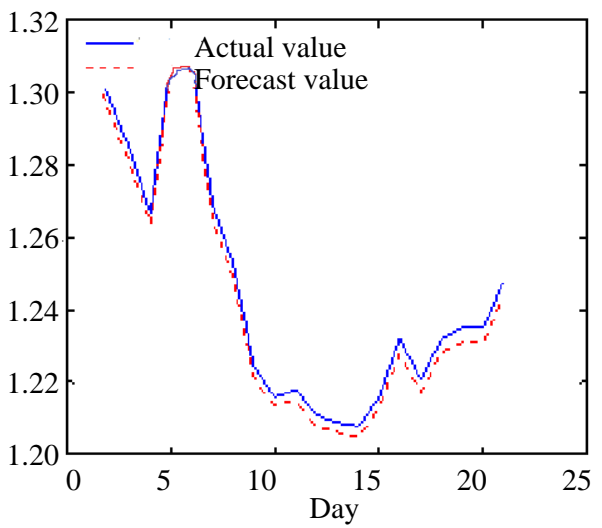

Fig. 8. Actual and forecast carbon price of case 4

TABLE II: THE RMSE, MAE, AND MAPE FOR ALL CASES

\begin{tabular}{|c|c|c|c|}
\hline Case & MAPE $(\%)$ & RMSE $(\%)$ & MAE \\
\hline 1 & 7.340 & 12.13 & 0.1589 \\
\hline 2 & 6.600 & 8.370 & 0.1633 \\
\hline 3 & 9.140 & 7.620 & 0.1301 \\
\hline 4 & 9.180 & 7.000 & 0.1146 \\
\hline
\end{tabular}

\section{B. Performance Test}

Table III shows the learning performance of ARBFN, RBFN, BPN and PNN for comparison in Case 2. The number of sets of training data and test data are 440 and 61 , respectively. Due to the regulation of learning rate, by ACO, ARBFN has a better accuracy than other methods do. The proposed ARBFN shows the better performance than do other ANNs with the same training data.

TABLE III: COMPARISON WITH ARBFN, RBFN, PNN AND BPN IN CASE 2

\begin{tabular}{|c|c|c|c|c|c|}
\hline Method & $\begin{array}{c}\text { Number of } \\
\text { training data }\end{array}$ & $\begin{array}{c}\text { Number } \\
\text { of test data }\end{array}$ & $\begin{array}{c}\text { MAPE } \\
(\%)\end{array}$ & RMSE & MAE \\
\hline ARBFN & 440 & 61 & 6.600 & 8.370 & 0.1633 \\
\hline RBFN & 440 & 61 & 11.5745 & 11.27 & 0.1842 \\
\hline PNN & 440 & 61 & 13.9234 & 14.189 & 0.1921 \\
\hline BPN & 440 & 61 & 9.9515 & 12.598 & 0.1751 \\
\hline
\end{tabular}

\section{CONCLUSION}

This paper is sucessfully proposed the ARBFN for 
forecasting the carbon price. RBFN is able to deal with varied and complicated relations between input and output data, and ACO helps with the appropriated regulation of learning rates. These tools help to improve the forecasting results. The actual data were used to demonstrate the performance of ARBFN. The RMSE, MAPE and MAE values were calculated for the seasons under study, and the results show that the ARBFN can closely track the spikes, which other methods cannot easily do. Compared with other popular ANNs, the ARBFN is shown to be efficient and accurate. It thus has been proved that ARBFN has the capability to produce superior results for volatile price forecasting.

\section{ACKNOWLEDGMENT}

Financial support for this work by the National Science Council of R.O.C. under contract number NSC100-2221-E-230-025 is gratefully appreciated.

\section{REFERENCES}

[1] US Congress, "Clean air act amendments of 1990," 1990.

[2] United Nations Framework Convention on Climate Change. (2011). [Online]. Available: http://unfccc.int/2860.php

[3] Green Energy JOBS. (2011). [Online]. Available: http://www.green-energy-jobs.net/

[4] A. Emilie, C. Julien, and C. Benoit, "Pieces Drivers and Structura Breaks in European Carbon Prices 2005-2007," Energy Policy, vol. 36 , no. 2, pp. 787-797, 2008.

[5] Z. H. Feng, L. L Zou, and Y. M. Wei, "Carbon price volatility: Evidence from EU ETS,” Applied Energy, vol. 88, no. 3, pp. 590-598, 2011.

[6] M. Hiroyuki and J. Wenjun, "An ANN-Based Risk Assessment Method for Carbon Pricing," Electricity Market, 5th International Conference on European, pp.1-6, 2008.

[7] M. Hiroyuki and J. Wenjun, "A risk Method for carbon price prediction with hybrid intelligent model in consideration of variable selection of graphical modeling," ICSET Conference, pp. 1019-1024, 2008.

[8] J. D. Wu and J. C. Liu, "A forecasting system for car fuel consumption using a radial basis function neural network," Expert Systems with Applications, vol. 39, no. 2, pp. 1883-1888, 2012.

[9] S. A. Kalogirou, "Applications of artificial neural networks for energy system," Applied Energy, vol. 67, no. 1-2, pp. 17-35, 2000.

[10] R. Ghazali, A. J. Hussain, and P. Liatsis, "Dynamic ridge polynomial neural network: forecasting the univariate non-stationary and stationary trading signals," Expert Systems with Applications, vol. 38 , no. 4, pp. 3765-3776, 2011

[11] O. Cortes, G. Urquiza, and J. A. Hernandez, "Optimization of operating conditions for compressor performance by means of neural network inverse," Applied Energy, vol. 86, no. 11, pp. 2487-2493, 2009.
[12] W. M. Lin, C. D. Yan, C. H. Lin, and M. T. Tsay, "A Fault Classification Method by RBF Neural Network with OLS Learning Procedure," IEEE Transactions on Power Delivery, vol. 16, no. 4, pp. 473-77, 2001

[13] M. Dorigo and L. M. Gambardella, "Ant colony system: a cooperative learning approach to the traveling salesman problem," IEEE Transactions on Evolutionary Computation, vol. 1, no. 1, pp. 53-66, 1997.

[14] R. J. Mullen, D. Monekosso, S. Barman, and P. Remagnino, “A review of ant algorithms," Expert Systems with Applications, vol. 36, no. 6, pp. 9608-9617, 2009.

[15] P. K. Sivagaminathan and S. R. Ramakrishnan, "A hybrid approach for feature subset selection using neural networks and ant colony optimization," Expert Systems with Applications, vol. 33, no. 1, pp 49-60, 2007.

[16] M. Pedemonte, S. Nesmachnow, and H. Cancela, "A survey on parallel ant colony optimization," Applied Soft Computing, vol. 11, no. 8, pp. 5181-97, 2011.

[17] K. Yakup, A. B. Melek, and O. K. Baykan, "Predicting direction of stock price index movement using artificial neural networks and support vector machines: The sample of the Istanbul Stock Exchange,' Expert Systems with Applications, vol. 38, no. 5, pp. 5311-5319, 2011.

[18] M. T. Tsai and C. W. Yan, "The influence of carbon dioxide trading scheme on economic dispatch of generators," Applied Energy, vol. 88, no. 12, pp. 4811-4816, 2011.

[19] A. I. Tolis and A. A. Rentizelas, “An impact assessment of electricity and emission allowances pricing in optimised expansion planning of power sector portfolios," Applied Energy, vol. 88, no. 11, pp. 3791-3806, 2011.

[20] W. M. Lin, H. J. Gow, and M. T. Tsai, "Electricity price forecasting using enhanced probability neural network," Energy Conversion and Management, vol. 51, no. 12, pp. 2707-2714, 2010.

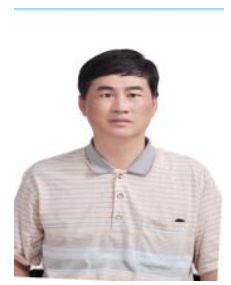

Ming-Tang Tsai was born on October $10^{\text {th }}, 1964$. He received BS-EE degree from Feng-Chia University in 1988. MS-EE and Ph.D. degree from the National Sun Yat-Sen University in 1990 and 1994. He has been with the Department of Electrical Engineering of Cheng-Shiu University., Taiwan, since 1994. He is interested in AI applications on distribution planning and power system operation.

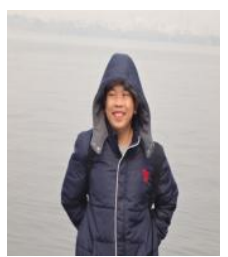

Yu-Teing Kuo was born on February $19^{\text {th }}, 1982 . \mathrm{He}$ received his MS-EE degree from the Cheng-Shiu University in 2012. Currently, he is pursuing his Ph.D. degree in the National Sun Yat-Sen University. His interests are Energy Management and Distribution Automatic System. 